\section{RMD Open}

Rheumatic \&

Musculoskeletal Diseases

\title{
Balancing on the edge: implications of a UK national audit of the use of BSR-BHPR guidelines for the diagnosis and management of polymyalgia rheumatica
}

\author{
Parthajit Das, ${ }^{1}$ Ash Samanta, ${ }^{1}$ Bhaskar Dasgupta ${ }^{2,3}$
}

To cite: Das P, Samanta A, Dasgupta B. Balancing on the edge: implications of a UK national audit of the use of BSR-BHPR guidelines for the diagnosis and management of polymyalgia rheumatica. RMD Open 2015;1:e000095. doi:10.1136/rmdopen-2015000095

- Prepublication history for this paper is available online. To view these files please visit the journal online (http://dx.doi.org/10.1136/ rmdopen-2015-000095).

Received 9 March 2015 Revised 14 May 2015 Accepted 10 June 2015

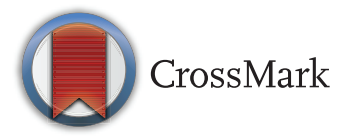

\section{${ }^{1}$ Department of}

Rheumatology, University Hospitals of Leicester NHS Trust, Leicester, UK

${ }^{2}$ Department of

Rheumatology, Southend University Hospital,

Southend, UK

${ }^{3}$ Anglia Ruskin, Essex, UK

Correspondence to Parthajit Das; parthajit. das21@gmail.com

\section{ABSTRACT}

Introduction: The British Society for Rheumatology and British Health Professionals in Rheumatology (BSR-BHPR) guidelines for management of polymyalgia rheumatica (PMR) were published in 2010, aiming to provide guidance for diagnosis, management and disease monitoring. A national study was conducted across multiple rheumatology units throughout the UK in order to study the level of adoption of guidelines in clinical practice.

Methods: This study was a retrospective analysis of patient records with a diagnosis of PMR from multiple centres across the UK. The primary objective was to explore the national compliance of PMR management according to BSR guidelines.

Results: We included 81 responses across 10 rheumatology units in the UK. The guideline core inclusion criteria were followed in more than $90 \%$ in making the diagnosis of PMR, but limited concordance was observed with respect to excluding PMR-mimics, the initial recommended glucocorticoid dosage $(74 \%)$, steroid taper $(41 \%)$, treatment of relapse $(76 \%)$, bone protection (84\%) and patient follow-up (43\%).

Conclusions: We observed a wide variation in clinical practice and limited adherence to BSR-BHPR guidelines in the UK. This study highlights the need for robust multilayered and multifaceted implementation strategies involving the providers and the consumers for apposite dissemination of guideline-based practice and consistency of care. We believe that the findings of this study have significant relevance for formulation and dissemination of PMR guidelines in the future.

\section{INTRODUCTION}

Polymyalgia rheumatica (PMR) is a common inflammatory condition affecting the elderly, and one of the commonest indications for long-term glucocorticoid therapy. ${ }^{1-3}$ Uncertainty related to diagnosis, disease

\section{Key messages}

What is already known on this subject?

- Polymyalgia rheumatic is a common medical condition managed in primary and secondary care by a wide variety of specialities. There is a wide variation of practice in terms of initiation, treatment duration, rate to tapering of glucocorticoid therapy and ancillary management. BSR-BHPR has published a guideline for diagnosis, management and monitoring of PMR, which is based on best evidence.

\section{What might this study add?}

- This study has observed limited concordance to guideline based practice in exclusion of PMR mimics, initiation, treatment duration and tapering of glucocorticoid therapy. This could be attributed to multiple factors such as multispeciality involvement, existence of plural guidelines for management of PMR in primary care, delayed presentation to secondary care, variability in patient response and lack of awareness of BSR-BHPR guideline.

\section{How might this impact on clinical practice?}

- This study highlighted the need for multi-layered and multi-faceted implementation strategies for raising awareness among the providers and the consumers for wider dissemination of guidelines.

monitoring and management of PMR can be challenging, thereby resulting in inconsistent clinical practice. ${ }^{4-6}$ There is need for evidencebased guidelines to reduce variations in practice across primary and secondary care as well as healthcare systems. These guidelines should aim at maximising positive patient outcomes while minimising the adverse effects of glucocorticoid therapy. It is apposite that our study 
is contemporaneous with European League Against Rheumatism (EULAR) and the American College of Rheumatology (ACR) PMR guidelines (manuscript submitted), the first transatlantic EULAR-ACR guidelines in rheumatology. Our study has implications for dissemination of these guidelines as well.

In 2010, the British Society for Rheumatology (BSR) and British Health Professionals in Rheumatology (BHPR) jointly published a guideline for management of PMR for the diagnosis, management and monitoring of disease. Similar guidelines were adopted by RCP and RCGP as well. ${ }^{7}$ This is relevant since a fair amount of PMR is diagnosed and treated in primary care and by non-rheumatologists. We have undertaken a study of the use of this guideline to ascertain the level of its adoption within clinical practice and to provide a critical commentary based on our findings.

\section{METHODS}

A national audit was conducted across several rheumatology centres in the UK to ascertain the degree of adoption of the BSR-BHPR PMR guidelines in practice. This audit, which formed the basis of the study, was a retrospective review of records of participants with a diagnosis of PMR. Fifteen leading rheumatology units with special interest in PMR across Scotland, England and Wales were invited and 10 centres participated.

Subjects were included only if they had PMR for at least 3 months and the diagnosis had been performed after 1 August 2011 and that this was the first presentation of PMR. A cut-off point of August 2011 was chosen as this was just over a year since the publication of the BSR-BHPR guideline, which we believe would be sufficient time for its embedding in clinical practice.

A questionnaire was designed to explore adherence to the BSR-BHPR guideline with respect to four domains agreed on by the authors to be of high clinical relevance. These were aimed at establishing the degree of clinician concordance with: (1) inclusion criteria; (2) exclusion of conditions resembling PMR ('PMR-mimics'); (3) glucocorticoid dosage and taper; and (4) follow-up, relapse and ancillary management (figures 1-3). Following further conceptual development based on feedback from a locally instituted pilot study, the questionnaire was placed on-line allowing access to participating centres.

Anonymised data were collected through a centralised portal of entry and subsequently analysed electronically to provide relevant metrics to inform results. Ethical approval was not necessary, as this study formed part of an audit exercise. External funding was not obtained.

\section{RESULTS}

Data of 81 participants with PMR submitted from 10 specialist centres in rheumatology in the UK were analysed. All were over 50 years of age and $67 \%$ were female.

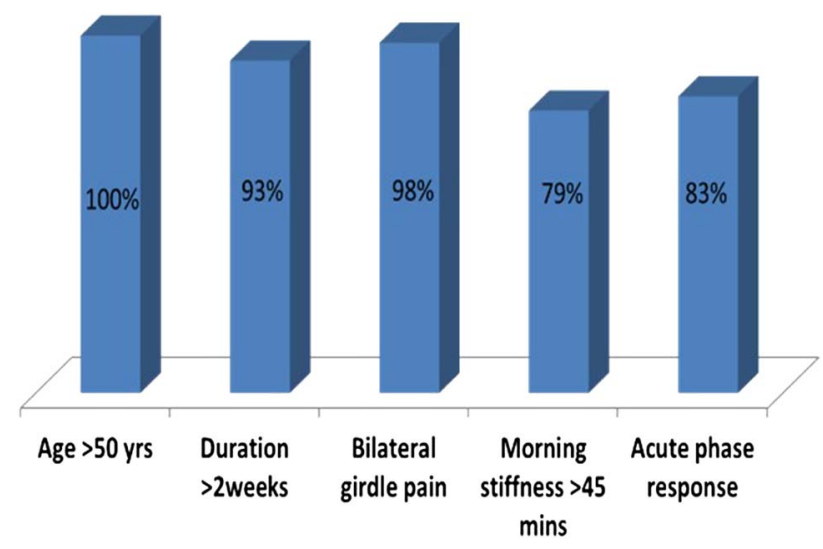

Figure 1 Inclusion criteria.

\section{Inclusion criteria}

Concordance for core inclusion criteria for diagnosis of PMR was: new onset pain (96.5\%); duration more than 2 weeks (93\%); bilateral shoulder or pelvic girdle aching (98\%); morning stiffness longer than 45 min (79\%); and evidence of acute phase response (83\%) (figure 1).

\section{Exclusion of PMR-mimics}

Routine investigations (full blood count, renal and liver functions, bone profile and inflammatory makers) were undertaken in $97 \%$ of subjects. There was considerable variation in practice regarding investigations for the exclusion of PMR-mimics: thyroid stimulating hormone $(72 \%)$, protein electrophoresis $(49 \%)$, Bence Jones proteins $(23 \%)$, rheumatoid factor $(65 \%)$, anti-cyclic citrullinated peptide (ACCP) (50\%), antinuclear antibody $(35 \%)$, chest X-ray $(57 \%)$.

\section{Glucocorticoid dosage and taper}

Seventy-four per cent of patients received an initial oral prednisolone dose compliant with the guidelines (15-20 mg/day). Ten per cent received a higher dose (25-40 mg). There was a preference for initial management of participants with oral prednisolone as opposed to corticosteroid injections. Taper of oral prednisolone was guideline-compliant in $41 \%$, and $30 \%$ of participants received oral glucocorticoid therapy for more than 2 years (figure 2).

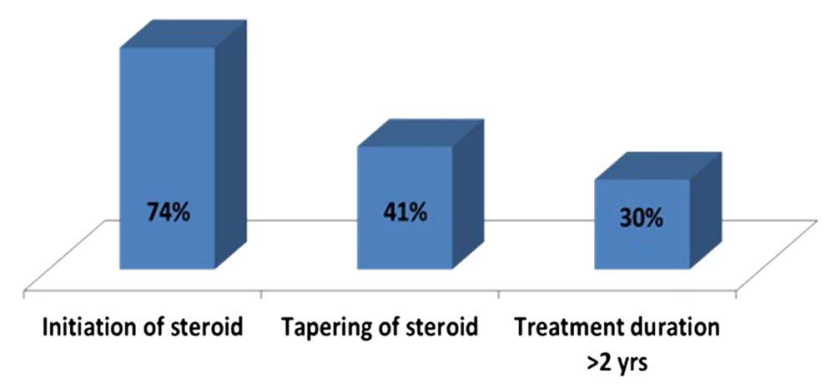

Figure 2 Glucocorticoid dosage and taper. 


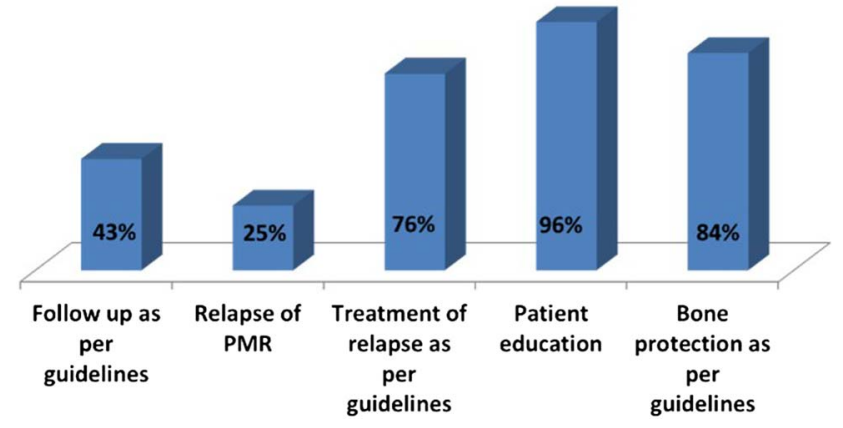

Figure 3 Follow-up, relapse and ancillary management.

Although bone protection was considered in more than $90 \%$ of patients, only $84 \%$ followed the guidelines. Almost all patients received calcium and vitamin D supplementation.

\section{Follow-up, relapse and ancillary management}

Forth three per cent adhered broadly to the follow-up regimen of the guideline.

Twenty-five per cent of patients experienced relapse of symptoms, mostly within the first 12 months. We observed wide differences in management of clinical relapses as well. Of these, $72 \%$ were treated with an incremental dose of prednisolone, $12 \%$ received intramuscular Depo-Medrone injection (between 40 and $120 \mathrm{mg}$ ) and disease modifying antirheumatic drugs (DMARDs) were considered only in $16 \%$ (generally only after the second relapse, as the 2010 BSR guidelines suggest). Overall, treatment of relapse was compliant in $76 \%$ of patients.

Ninety six were provided with patient education (counselling from medical practitioners and specialist nurses in 93\%) and $84 \%$ received bone protection (figure 3).

\section{DISCUSSION}

This is the only national study undertaking post hoc scrutiny of the use of BSR-BHPR guidelines for PMR. The guideline criteria were followed in $90 \%$ or over for making the diagnosis of PMR, but limited concordance was observed with respect to excluding PMR-mimics and the initial recommended low to moderate dose of glucocorticoid. At least $10 \%$ of patients received higher doses with a tapering regime non-aligned to guideline recommendations. In relapse of PMR, the use of DMARDs as adjuvant therapy was considered in a minority and at a late stage. However, this is consistent with 2010 BSR guidelines although the 2015 EULAR ACR guidelines encourage early use of DMARD with severe disease or high number of risk factors for glucocorticoid AEs. There was limited evidence for bone and gastro protection. Although patient education was declared as having been undertaken, the extent and quality of this remains unclear. These findings show that more than 3 years since publication, adherence to the guideline in clinical practice is at best somewhat loose.

We acknowledge that this study was undertaken within a secondary care setting. Yet the results apply equally to primary care, since the overarching principles for diagnosis and management for PMR (as set out in the EULAR ACR guidelines) remain the same. Evidence exists that of all rheumatological conditions, the highest discordance between primary and secondary care exists in the diagnosis of PMR. ${ }^{6}$ Difficulty in establishing the diagnosis of PMR in primary care can lead to substantial utilisation of healthcare resources and increased cost. A retrospective chart review of 123 PMR patients referred to a tertiary Canadian rheumatology clinic demonstrated that only $24 \%$ of patients were correctly identified as PMR by family physicians at time of referral. $^{8}$ The mean cost of investigations to establish a diagnosis prior to referral was 3.8 times higher than the consensus estimate cost of diagnosing uncomplicated PMR advocated by local rheumatologists.

The above study suggests that educational programmes on the guidelines (presentation, evaluation, timely referral, management, steroid tapering of patients with PMR) are needed to assist in effective diagnosis and also makes the economic case for early referral to the rheumatologist for confirmation of the diagnosis in PMR. This is especially relevant since there is as yet no 'gold standard' test for this diagnosis. The shibboleth of current wisdom - a brisk response to steroids (often instituted on the basis of 'clinical intuition') - has been shown to be without evidence. ${ }^{9}$ Sadly, PMR is usually referred late to secondary care due to diagnostic uncertainty, steroid-induced complications or inability to reduce steroids-factors that reflect in non-adherence to guidelines. Steroid tapering can be challenging, and needs to be predicated on a balance between adverse events and disease activity. Owing to the above reasons, we feel that poor compliance with these guidelines reflects not only technical infringements, but also practice that may adversely affect outcomes. We suggest a future longitudinal observational study of outcomes (not within the remit of the present study) with adequate representation across primary and secondary care, to confirm the value of adherence to guidelines in PMR.

Limitations of this study include a small number of centres and patients, short study duration, self-reported data (although based on record review) and lack of single point of care due to involvement of multiple specialities (primary care, general internists, care of the elderly and rheumatologists). Nevertheless, our findings highlight the areas of limited guideline concordance and the relevance of this for further guideline implementation.

A number of hurdles may deter their use of the BSR-BHPR PMR guideline. The guideline has been produced by specialist opinion leaders, general practitioners GPs and frontline clinicians (rheumatologists, GPs, ophthalmologists, internists, methodologists), but it 
requires training to develop the expertise to assess PMR diagnosis and management within heterogeneous presentations. Older people with PMR frequently have comorbidities that impact on treatment, thereby leading to valid departure from the guideline. There is need for active empowerment of patients to assist in the wider dissemination of the guideline and the newly formed PMRGCAuk, a charity dedicated to PMR and giant cell arteritis (GCA), has this as its main objective. What is of some concern is that shift of long-term condition management to the community may adversely affect adherence to these guidelines.

The usefulness of the EULAR ACR PMR recommendations in achieving high quality care for participants with PMR will only be through confirmation of its acceptance in clinical practice. It will be important to have a feasible audit tool as part of the new guidelines. We hope that our report draws special attention to the hurdles facing the implementation of PMR guidelines; especially since polymyalgia is now viewed as part of the spectrum of late onset inflammatory rheumatological diseases.

Acknowledgements The authors acknowledge the help and support from the Clinical Audit Team at University Hospitals of Leicester NHS Trust for their help in the analysis of the data, and all the clinicians across several centres in the UK for participating in this national audit. The authors are grateful to the three anonymous referees for their constructive comments on an earlier version of this paper.

Contributors All the authors including PD, AS and BD were actively involved in the project. The listed authors fulfilled the ICMJE guidelines for authorship including conceptual design of the study, the recruitment of participating centres, data analysis and interpretation, drafting the article, and revising and final approval of this paper.

Funding We have not received funding from any national or regional organisations or any pharmaceutical companies.

Competing interests None declared.
Ethics approval This study was based on a national audit-based exercise on diagnosis and management of PMR. The audit included retrospective review of medical records of participants with a diagnosis of PMR across multiple centres in the UK through an online portal. Patient identifiable details were anonymised. Discussion with the senior clinicians and clinical governance teams confirmed that ethical approval was not required as this study was part of an audit exercise.

Provenance and peer review Not commissioned; externally peer reviewed.

Data sharing statement No additional data are available.

Open Access This is an Open Access article distributed in accordance with the Creative Commons Attribution Non Commercial (CC BY-NC 4.0) license, which permits others to distribute, remix, adapt, build upon this work noncommercially, and license their derivative works on different terms, provided the original work is properly cited and the use is non-commercial. See: http:// creativecommons.org/licenses/by-nc/4.0/

\section{REFERENCES}

1. Dasgupta B, Borg FA, Hassan N, et al. BSR and BHPR guidelines for the management of polymyalgia rheumatic. Rheumatology 2010;49:186-90.

2. Weyand CM, Goronzy JJ. Giant-cell arteritis and polymyalgia rheumatica. Ann Intern Med 2003;139:505-15.

3. Doran MF, Crowson CS, O'Fallon WM, et al. Trends in the incidence of polymyalgia rheumatica over a 30year period in Olmsted County, Minnesota, USA. J Rheumatol 2002;29:1694-7.

4. Chakravarty K, Elgabani SH, Scott DG, et al. A district audit on the management of polymyalgia rheumatica and giant cell arteritis. $\mathrm{Br} \mathrm{J}$ Rheumatol 1994;33:152-6.

5. Gamez-Nava JI, Gonzalez-Lopez L, Davis P, et al. Referral and diagnosis of common rheumatic diseases by primary care physicians. Br J Rheumatol 1998;37:1215-9.

6. Bahlas S, Ramos-Remus C, Davis P. Utilisation and costs of investigations, and accuracy of diagnosis of polymyalgia rheumatica by family physicians. Clin Rheumatol 2000;19:278-80.

7. Concise Guidance: Diagnosis and management of polymyalgia rheumatica B Dasgupta, Guidelines Development Group. Clinical Medicine 2010;10:270-4.

8. Brooks RC, McGee SR. Diagnostic dilemmas in polymyalgia rheumatica. Arch Int Med 1997;157:162-8.

9. Dasgupta B, Cimmino MA, Maradit-Kremers H, et al. 2012 provisional classification criteria for polymyalgia rheumatica: a European League Against Rheumatism/American College of Rheumatology collaborative initiative. Ann Rheum Dis 2012;71:484-92. 